\title{
Inkjet Printed Nanohydrogel Coated Carbon Nanotubes Electrodes For Matrix Independent Sensing
}

\author{
Andreas Lesch, ${ }^{\dagger}$ Fernando Cortés-Salazar, $^{\dagger}$ Véronique Amstutz, $^{\dagger}$ Philippe Tacchini, $^{\dagger}$ \\ and Hubert H. Girault*, ${ }^{\dagger}$ \\ ${ }^{\dagger}$ Laboratoire d’Electrochimie Physique et Analytique, École Polytechnique Fédérale de Lausanne, Station 6, CH-1015 Lausanne, \\ Switzerland \\ ${ }^{\ddagger}$ Edel for Life, PSE-B/EPFL, CH-1015 Lausanne, Switzerland
}

\section{Supporting Information}

ABSTRACT: Polyacrylamide (PA) based hydrogels are used in several applications including polyacrylamide gel electrophoresis and sensing devices. Homogeneous and compact PA films can be prepared based on chemical or photopolymerization processes. However, the accurate and reproducible coating of substrates with nanohydrogel patterns is challenging due to the in situ polymerization and deposition requirements. Herein, we report an inkjet printing (IJP) concept with simultaneously performed UV photopolymerization of a specifically prepared acrylamide $/ N, N^{\prime}$-methylenebis(acrylamide) containing ink. A prepolymerization step of the hydrogel precursor molecules was implemented in the ink formulation protocol to adjust the viscosity of the ink and to enhance the rate of polymerization during printing. After the optimization of the printing parameters, a nanometer thin PA hydrogel coating with well distributed nanopores was achieved on top of a stand-alone carbon nanotubes (CNTs) pattern. Batches of fully inkjet printed PA/CNT modified electrodes were prepared that showed outstanding improvements for the electrochemical detection of antioxidants in complex matrices such as untreated orange juice and red wine samples thanks to the properties of the PA coating.

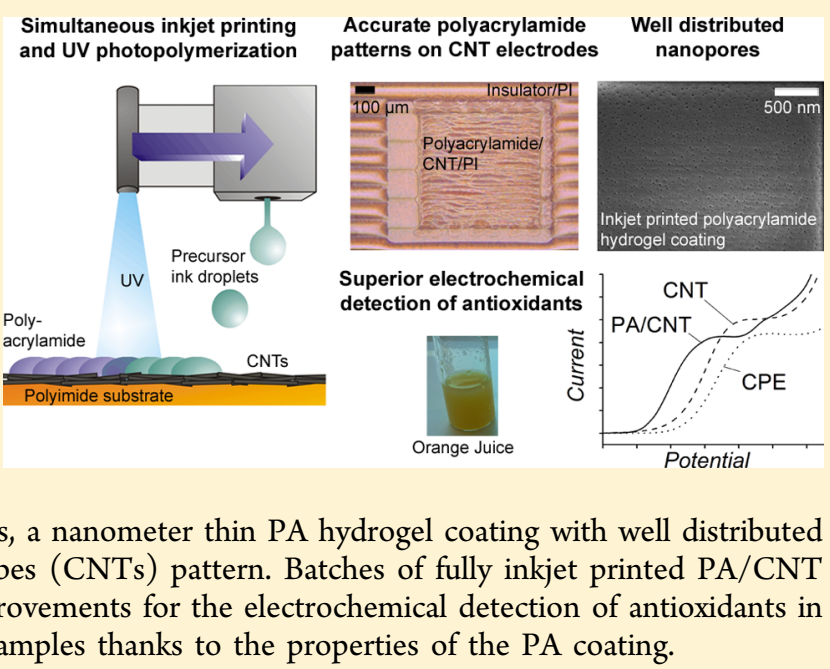

$\mathrm{P}$ olyacrylamide (PA) is a hygroscopic material that forms a hydrogel when wetted with water. It can be of linear or cross-linked nature and its hydrophilic properties and controllable nanopore size make it suitable for many applications including polyacrylamide gel electrophoresis (PAGE), ${ }^{1}$ flocculation in water treatment, ${ }^{2}$ enhanced oil recovery process ${ }^{3}$ or by providing a porous matrix to bind catalytically active compounds close to an electrode surface. ${ }^{4-9}$ The latter profits from both the binding properties and the porous structure of the PA layer, allowing the mass transport of analytes toward the embedded active sites and the electrode surface. However, up to now, PA has not been considered as a pure chemically modifying film for electrochemical sensors, although such chemically modified electrodes (CMEs) principally could profit from the hydrogel's properties. CMEs represent one of the main research fields in electrochemistry with many applications including analytical chemistry, ${ }^{10,11}$ solar energy conversion, ${ }^{12,13}$ electrochromic display devices ${ }^{14}$ and molecular electronic devices. ${ }^{15}$ Generally, many efforts have been made to develop CMEs with the goal of improving the sensitivity, selectivity and reproducibility under real sample conditions or of protecting electrodes from passivation. ${ }^{10,11,16-19}$ In particular, polymer based CMEs offer many opportunities due to the variety of preparation routes and potentially enhanced electrode functionalities. $^{20}$ Generally, thin polymeric layers can be prepared using solutions of either the dissolved polymer or the corresponding monomer. Polymer containing solutions can be drop-casted, dip-coated, electrodeposited or covalently attached. Alternatively, dissolved monomers can be polymerized in situ on the electrode surface by electrochemical, photochemical or thermal means. CMEs complemented with pure PA films have only been reported previously by Lange et al., who prepared dry graphite-PA-composite mixtures that were packed inside a Teflon sleeve, back connected with a carbon strip and used as sensing CMEs in small sample volumes. $^{21,22}$ The authors terminated the polymerization process of their acrylamide containing solution before the gelation was finished in order to obtain a viscous PA composition mixable with graphite powder. ${ }^{21}$ The main drawback of the reported PA sensors was the low mechanical stability, which is compromised by the swelling process of the composite material that detached from the electrode already after few hours or when the $\mathrm{pH}$ of the media was smaller than 7 or larger than $9 .^{21}$

One additional reason for the low interest in PA hydrogels as pure modifying films could be due to the limited fabrication

Received: September 23, 2014

Accepted: December 20, 2014

Published: January 2, 2015 
routes of thin homogeneous films on electrodes. PA hydrogels are generally 1-2 $\mathrm{mm}$ thick and are used in PAGE where proteins or nucleic acids can be separated by their molecular masses or their isoelectric point in the presence of an electric field. ${ }^{23,24}$ Such PA gels are usually created by chemical or UV photopolymerization in aqueous solutions containing the monomer acrylamide and the cross-linker $N, N^{\prime}$-methylenebis(acrylamide) ("bis"). The underlying reaction mechanism is a free radical copolymerization that is started by radical initiators such as ammonium persulfate or riboflavin. $N, N, N^{\prime}, N^{\prime}$ tetramethylethylenediamine (TEMED) is commonly added as a catalyst to increase the rate of polymerization. The characteristic properties of the resulting hydrogel, such as the porosity, depend on the polymerization conditions (temperature, oxygen content, $\mathrm{pH}$, etc.), on the concentrations of catalyst, initiator, monomer and cross-linker and on the acrylamide/bis ratio (usually between 2.6 and 5\% C).

Ultrathin layers of PA hydrogels, in literature, are usually defined as around $120-360 \mu \mathrm{m}$ thick, and have shown an improved resolution for isoelectric focusing (IEF) based PAGE. $^{25,26}$ Such ultrathin films can be prepared in polymerization chambers consisting of two parallel glass slides separated only by a thin spacer that defines the distance between the glass plates and thus the obtained gel thickness. Normally, ultrathin hydrogels are accompanied by a cellophane sheet in order to provide adhesion and mechanical stability of the gel. In an alternative approach, solutions containing dispersed macromolecular PA particles have been used to prepare nanometer thin films on silica by spin coating. ${ }^{27}$ However, this approach results in an assembly of PA macromolecules rather than in a polymerized, covalently connected network.

PA films thinner than $10 \mathrm{~nm}$ can be generated by surfaceconfined living or atom-transfer radical polymerization, ${ }^{28-31}$ for instance, as coatings on the inner surface of silica capillaries in capillary electrophoresis to reduce the capillary's surface potential and the electro-osmotic flow for an enhanced protein separation $^{28}$ or, alternatively, to increase the hydrophilicity of poly(dimethylsiloxane) microchannels for rapid electrophoretic protein separation. ${ }^{30}$

Nowadays, digital printing technologies such as inkjet printing (IJP) gain importance for the deposition of functional thin films. ${ }^{32,33}$ IJP has been successfully employed for the fabrication of organic thin film transistors (OTFTs), organic light emitting diodes (OLEDs), organic solar cells, sensing devices and biomaterials. ${ }^{34,35}$ By IJP, a defined functionality is created with a micrometer resolution on many kinds of substrates by the controlled contact- and maskless deposition of picoliter droplets. Several postprocessing techniques such as sintering and UV photopolymerization transform the initial ink components into a functional film by evaporation of the solvent and additives and by polymerization, respectively. Inkjet printing allows testing, prototyping and is up-scalable to the industrial level. However, for stable and reproducible printing of functional inks, certain requirements regarding the ink properties need to be fulfilled, such as viscosity, surface tension, size of nanoparticles and shelf life. Certainly, those characteristics depend on the printhead, but generally, the typical composition of acrylamide/bis solutions is not suitable for most IJP concepts. Moreover, a simultaneous polymerization, either chemically or photochemically, is required to obtain a homogeneous, compact and highly resolved PA film. Therefore, reported approaches for IJP of PA are only based on the deposition of arrays of individual, separated droplets. For instance, Liu et al. printed an acrylamide/bis solution whose viscosity has been adjusted by the addition of glycerol. ${ }^{36}$ The UV photopolymerization had to be performed subsequently for $8 \mathrm{~min}$ as a relatively slow postprocess compared to the printing frequency. Furthermore, a polyethylene glycol (PEG) modification of the silica substrate was required to cross-link the gel to the silica substrate. Recently, Wang et al. reported the IJP without polymerization step of an ink with PA macromolecules to construct a light guide plate that consisted of individual PA spots. $^{37}$

Herein, we demonstrate reproducible simultaneous IJP and complete UV photopolymerization of aqueous acrylamide/bis ink formulations containing a photoinitiator and a catalyst. The result is a compact and mechanically stable nanoporous PA coating. The surface tension of the aqueous ink was adjusted by adding a nonionic surfactant and the viscosity of the ink was increased using a well-controllable prepolymerization approach avoiding the addition of unwanted chemicals to the ink. We applied the printing strategy to fully inkjet printed, disposable stand-alone carbon nanotubes (CNTs) amperometric sensors, which have recently been established for antioxidant (AO) detection in relevant biological fluids. ${ }^{38}$ We demonstrate that the PA hydrogel coating provides an enhanced response to the CNT electrodes independent from matrix effects that are usually induced by complex samples such as undiluted red wine or orange juice. Moreover, the PA/CNT electrodes show outstanding characteristics in terms of reproducibility and selectivity compared to screen printed carbon paste and bare CNT electrodes even in undiluted samples.

\section{EXPERIMENTAL SECTION}

Materials. Ferrocenemethanol (FcMeOH; Sigma-Aldrich), L(+)-Ascorbic acid (AA; Riedel de Haën), gallic acid (GA; Alfa Aesar), acetic acid (Merck Millipore), sodium acetate (Fluka) potassium chloride ( $\mathrm{KCl}$; Sigma-Aldrich), acrylamide/bis solution (40\% (w/v), 2.6\% C, Serva/Promega), TEMED (Sigma-Aldrich) and riboflavin (Fluka) were used as received and were of analytical grade. Deionized (DI) water was produced by a Milli-Q plus 185 model (Merck Millipore). Jettable nanosilver EMD5603 (w/w 20\%), jettable insulator EMD6201 (both Sun Chemical) and the CNT dispersion CNTRENE (Brewer Science) were used as inks for IJP. As specified by the manufacturer, the CNTs are mostly doublewalled (DW)CNTs with an unknown amount of single-walled (SW)CNTs with lengths $(763.08 \pm 595.16) \mathrm{nm}$ and diameters $(1.56 \pm 0.56) \mathrm{nm}$. The CNTs were functionalized by the manufacturer with 1-pyrenemethylamine-3,6,8-trisulfonic acid groups to ensure a stable dispersion free of aggregates. Screen printed carbon paste electrodes (CPE) were provided by Edelfor-Life. Orange juice and red wine samples were purchased in a local supermarket. The fresh orange juice contained pulp and fibers and the red wine was a Swiss Dôle from the Canton Valais that is mainly composed of Pinot Noir and Gamay.

Acrylamide/Bis Ink Formulation. Gel precursor solutions for IJP were prepared in two steps: (i) by a prepolymerization and (ii) by formulating the final ink. For the UV light induced prepolymerization, an aqueous solution containing acrylamide/ bis $(7.88 \%(\mathrm{w} / \mathrm{v}), 2.6 \% \mathrm{C})$, Riboflavin $(1.14 \mu \mathrm{g} / \mathrm{mL})$ and TEMED $(0.0057 \%(\mathrm{w} / \mathrm{v}))$ was stirred $(500 \mathrm{rpm})$ and exposed for $1 \mathrm{~h}$ to a Xe arc lamp (LOT-Oriel, model 66021) with an adjusted power of $600 \mathrm{~W}$ and a liquid filter to exclude IR radiation. Subsequently, photoinitiator, catalyzer and surfactant were added to this mixture to achieve a final ink composition of 
$4.6 \mu \mathrm{g} / \mathrm{mL}$ riboflavin, $0.14 \%(\mathrm{w} / \mathrm{v})$ TEMED and $0.65 \mu \mathrm{L} / \mathrm{mL}$ Triton-X 100, respectively. The surface tension of the self-made inks was measured using a Drop Shape Analyzer DSA100 (Krüss), and the viscosity was determined with a SV-1A series viscometer (A\&D Instruments Limited).

Preparation of PA/CNT Electrodes. PA/CNT electrodes were prepared by a four layer IJP process using the drop-ondemand Dimatix materials printer DMP-2831 and a $10 \mathrm{pL}$ droplet volume cartridge (Dimatix Fujifilm). A custom-made modification of the IJP allowed mounting of a liquid light guide connected to an Omnicure S2000 mercury UV lamp (Lumen Dynamics). Because the Dimatix software allows the opening of the light guide shutter during printing, a photopolymerization just slightly after the deposition of droplets is realized. The first three layers, i.e., a $\mathrm{Ag}$ pattern for the electrical connection, CNTs as active electrode material and an insulating layer to define accurately the active CNT electrode area $\left(0.36 \mathrm{~mm}^{2}\right)$, were prepared on $125 \mu \mathrm{m}$ thick Kapton HN sheets (polyimide (PI); Goodfellow) as reported previously. ${ }^{38}$ All printing parameters like waveform, jetting frequency, cartridge and substrate temperature, etc. were optimized for each ink.

Reproducible PA layers were obtained in the final IJP step using the precursor solution that was optimized for accurate printing and fast simultaneous UV photopolymerization. Printing parameters such as drop spacing and frequency were adjusted for one nozzle use, and up to four layers of PA were deposited directly on top of the CNT electrodes, covering the entire active electrode area. After washing with DI water, no further treatment was required prior to use.

Optical Characterization Methods. The printed patterns were investigated using laser scanning microscopy in reflection mode with a Keyence VK 8700 (Keyence) and high-resolution scanning electron microscopy (HR SEM) using a SEM MERLIN composed of a GEMINI II column (Zeiss). Before HR SEM, a PA/CNT electrode was placed inside an Eppendorf tube and completely covered by $100 \mu \mathrm{L}$ of $\mathrm{H}_{2} \mathrm{O}$. The sample was shock-frozen by immersing the Eppendorf tube for $10 \mathrm{~min}$ into liquid nitrogen. Immediately after, the tube was transferred into a lyophilizer at a pressure of $0.1 \mathrm{mbar}$ and at a condensation temperature of $-87{ }^{\circ} \mathrm{C}$ for freeze-drying for 4 $h$. The freeze-dried sample was then analyzed by HR SEM, and the obtained pictures represent the real hydrogel structure when immersed in aqueous solution. The pore size distribution was determined using ImageJ software $1.48 \mathrm{v}$ (W. Rasband, National Institutes of Health, USA).

Electrochemical Measurements. Cyclic voltammetry (CV) and linear sweep voltammetry (LSV) were carried out with a potentiostat Autolab PGSTAT101 (Metrohm) in a three-electrode arrangement using the CNT or the PA/CNT electrodes as working electrodes, a $\mathrm{Pt}$ wire as the counter electrode and either a $\mathrm{Ag} / \mathrm{AgCl} / 1 \mathrm{M} \mathrm{KCl}$ electrode as the reference electrode (RE) or a $\mathrm{Ag}$ wire as the quasi-RE ( $\mathrm{QRE}$ ). All measurements were performed under ambient conditions at room temperature without any pretreatment of the CNT electrodes.

\section{RESULTS AND DISCUSSION}

Ink Formulation. To generate highly accurate patterns of PA films by IJP and simultaneous UV photopolymerization of acrylamide/bis containing inks (reaction schemes in the Supporting Information, SI-1), two main issues have to be overcome: (i) the ink has to be printable and (ii) a rapid UV photopolymerization has to be realized to form a homogeneous
PA hydrogel film. Typical formulations for standard preparations of PAGE hydrogels based on UV photopolymerization contain $3-30 \%(\mathrm{w} / \mathrm{v})$ of total monomer and cross-linker (acrylamide + bis), $5 \mu \mathrm{g} \cdot \mathrm{mL}^{-1}$ initiator like riboflavin and $0.05 \%$ catalyst (TEMED). This leads to a surface tension of $\sim 72.8 \mathrm{mN} \cdot \mathrm{m}^{-1}$ and a viscosity of $\sim 1 \mathrm{mPa} \cdot \mathrm{s}$ due to the high water content in the ink. Because the cartridges of the DMP2831 require inks with a viscosity between 2 and $30 \mathrm{mPa} \cdot \mathrm{s}$ (ideally $8-12 \mathrm{mPa} \cdot \mathrm{s}$ ) and a surface tension between 20 and 40 $\mathrm{mN} \cdot \mathrm{m}^{-1}$ (ideally $28-33 \mathrm{mN} \cdot \mathrm{m}^{-1}$ ), stable and reproducible IJP of the typical PAGE formulations is hardly possible. The surface tension can be lowered to $32 \mathrm{mN} \cdot \mathrm{m}^{-1}$ by using small amounts of the nonionic surfactant Triton X-100, but the adjustment of the viscosity is not an easy task. One strategy to increase the viscosity is the use of highly viscous organic liquids such as ethylene glycol (EG), but this can influence the UV photopolymerization process and it will require additional treatments (e.g., washing or thermal treatment) to remove the EG after printing. Another strategy could be the addition of $50 \%(\mathrm{w} / \mathrm{v})$ macromolecular PA solutions (average molar masses > $1000 \mathrm{~g} \mathrm{~mol}^{-1}$ ) that are characterized by high viscosities. However, such an approach reduces the accuracy of the pore size in the inkjet printed PA layer and the procedure might also lack in covalent linking of the PA macromolecules to the UV polymerized PA structure, which will prevent the formation of a compact film (Supporting Information, SI-5). Therefore, we developed a strategy to overcome both issues by performing a controlled pre-UV-photopolymerization at room temperature using a 4-fold reduced amount of photoinitiator and about 25 times reduced concentration of catalyst compared to the standard composition for, e.g., PAGE gels. The polymerization process in the initial slightly yellowish composition takes place under stirring to homogenize the solution during the reaction. The reaction comes to a stop due to the complete consumption of radical sources and leads to a colorless liquid with an increased viscosity that is suitable for IJP and far from gelation. After the addition of optimized amounts of photoinitiator, catalyst and surfactant, the measured viscosity is $1.80-2.00 \mathrm{mPa} \cdot \mathrm{s}$ compared to $1.04 \mathrm{mPa} \cdot \mathrm{s}$ without prepolymerization. The resulting ink is stable and does not continue to polymerize when protected from UV light. A prepolymerization process with higher concentration of catalyst and/or photoinitiator leads to a more progressed prepolymerization and a viscosity larger than $2.00 \mathrm{mPa} \cdot \mathrm{s}$ resulted already in a fiber-like droplet formation (Supporting Information, SI-2) or in a complete blocking of the nozzles. A new waveform was created (Supporting Information, SI-3), characterized mainly by a relatively long firing period, to enable a proper jetting of the developed final ink. Stable droplet formation could be obtained as shown for six consecutive nozzles in Figure 1a.

Multilayer Inkjet Printing and Optical Characterization. Generally, the preparation of microarray structures by the IJP of locally separated droplets is quite easy as long as the ink allows stable jetting whereas the printing of lines and defined patterns is far more challenging due to the required appropriate overlapping of adjacent droplets. Depending on the surface energy of the substrate, the deposited ink droplets can merge on the surface to form significantly larger droplets rather than staying in their initial locations. This results in a loss of resolution of the desired patterns, incomplete gel formation and/or precipitation of remaining reagents as salt due to the fast water evaporation at the thin liquid film. On the basis of the design of stand-alone CNT electrodes composed of three 


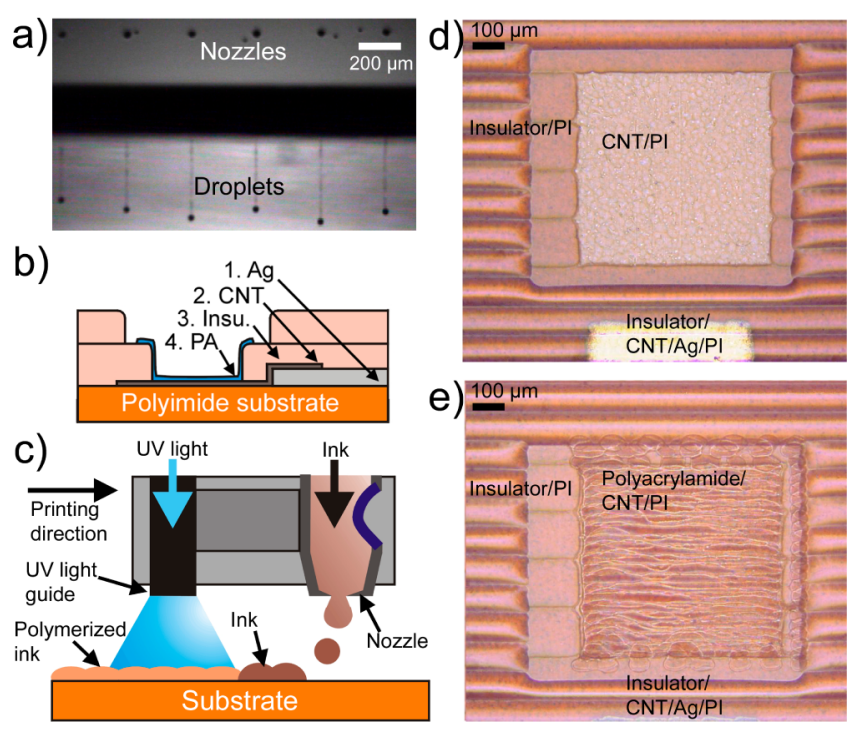

Figure 1. (a) Droplet formation recorded with the Dimatix drop watcher camera and using six nozzles simultaneously and applying the developed waveform (Supporting Information, SI-3). (b) Schematic representation of fully inkjet printed PA/CNT electrodes based on subsequent printing of $\mathrm{Ag}, \mathrm{CNT}$, insulator and PA. (c) Schematic representation of simultaneous IJP and UV photopolymerization. Optical micrographs of (d) fully inkjet printed CNT and (e) PA/CNT electrode.

consecutively printed materials (i.e., Ag, CNT and insulator), ${ }^{38}$ a PA deposition was carried out as the fourth printing sequence (Figure 1b). The simultaneous UV photopolymerization was carried out just slightly after the droplet deposition thanks to the integrated UV light guide (Figure 1c). The design of the PA pattern was defined slightly larger $\left(0.49 \mathrm{~mm}^{2}\right)$ than the active CNT electrode area $\left(0.36 \mathrm{~mm}^{2}\right.$, Figure 1d) to ensure a complete coverage of the active sensor surface. Indeed, by using one nozzle, a drop spacing of $30 \mu \mathrm{m}$ and a maximum jetting frequency of $1.3 \mathrm{kHz}$ well-defined PA patterns of $0.7 \mathrm{~mm} \times 0.7$ $\mathrm{mm}$ size could be printed (Figure 1e). Two inkjet printed layers (IJPL) of PA were required in order to obtain complete coverage because few uncovered regions can be obtained in a single layer (Supporting Information, SI-4). The final 2 IJPL PA film is compact, completely polymerized and well attached, which was confirmed by wetting with DI water. Even flushing with DI water did not detach the film, which would be the case for a not completely polymerized PA film (Supporting Information, SI-5).

In addition to the adjustment of the viscosity, the prepolymerized ink showed also an increased rate of acrylamide/bis polymerization in comparison to the nonpretreated ink formulations. The reason for such result might be explained as the polymerization can be continued from the vinyl groups available in the pregenerated PA molecules due to the present bis(acrylamide) (Supporting Information, SI-1), which could provide a faster cross-linking and shorter time to reach the gelation point. The substrate selection plays also a major role for the fast and efficient polymerization because no PA could be formed on non-treated polyethylene terephthalate (PET) using the same parameters as on pure PI and bare CNT electrodes (Supporting Information, SI-6).

Figure 2a shows HR SEMs of the fully inkjet printed compact bare $\mathrm{CNT}$ and Figure $2 \mathrm{~b}$ of $\mathrm{PA} / \mathrm{CNT}$ electrodes (more HR SEMs in the Supporting Information, SI-7). Indeed,

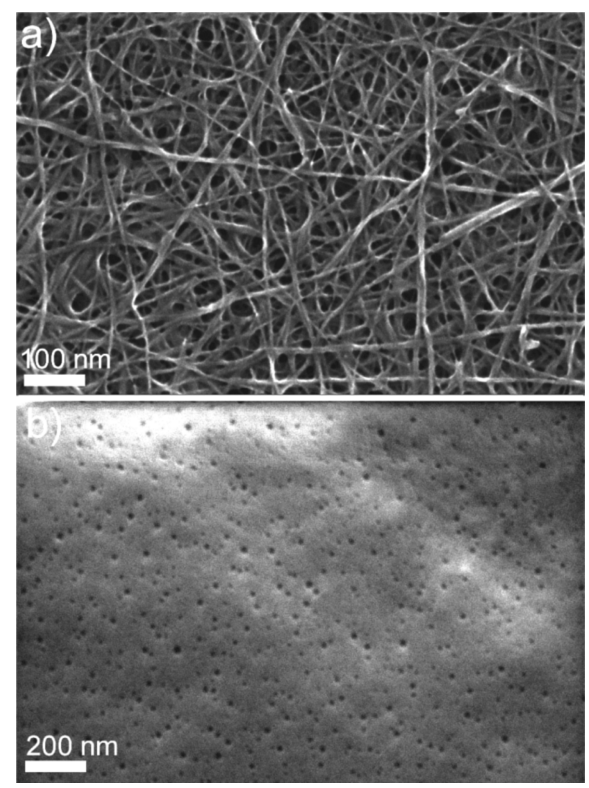

Figure 2. HR SEM of (a) bare CNT electrodes and (b) PA on CNT electrodes.

the pores in the hydrogel structure are well distributed over the surface and have diameters of $(13.3 \pm 9.1) \mathrm{nm}$ (Supporting Information, SI-8). The estimated thickness of the PA film is between few nanometers up to $1 \mu \mathrm{m}$ (Supporting Information, SI-9). This variation originates from a coffee ring effect drying behavior, ${ }^{39,40}$ resulting in a higher gel thickness at the rims compared to the center of printed lines. Nevertheless, as it can be seen in Figures $1 \mathrm{e}$ and $2 \mathrm{~b}$, a 2 IJPL PA film covers completely the CNT structure.

Electrochemical Characterization. The electrochemical response of the $\mathrm{PA} / \mathrm{CNT}$ electrodes was tested by $\mathrm{CV}$ in solutions containing $\mathrm{FcMeOH}$ as redox active species (Figure $3)$. Three different fresh sensors for each type of CNT, PA/ CNT (i.e., 2 IJPL PA) and $(2 \times$ PA)/CNT (i.e., 4 IJPL PA) electrodes were employed. In all cases, the oxidation and reduction waves of the $\mathrm{FcMeOH} / \mathrm{FcMeOH}^{+}$redox couple were clearly identified, meaning that the CNT electrode surface is accessible through the nanoporous structure of the PA film. The mass transport of $\mathrm{FcMeOH}$ through the hydrogel is controlled by semi-infinite linear diffusion due to the overlapping of the diffusion layers of all individual pores. CVs with multiple cycles demonstrate that the hydrogel shows well overlapping continuous cycles over time (Supporting Information, SI-10).

This indicates that the PA hydrogel can be applied immediately after the immersion in electrolyte solution and does not require a pretreatment step such as prewetting. For each electrode type, almost identical CVs were obtained using different sensors, demonstrating the equality of the active electrode areas and, consequently, the reproducibility of the multilayer IJP process. The height of the anodic peak currents $I_{\mathrm{pa}}$ in the CVs recorded with a scan rate of $100 \mathrm{mV} \cdot \mathrm{s}^{-1}$ vary by $0.2 \%$ for the CNT, $0.9 \%$ for the PA/CNT and $3.3 \%$ for the $(2$ $\times$ PA)/CNT electrodes. This shows that the reproducibility of the sensors is slightly lowered with increasing number of IJPLs of PA. Furthermore, the electrode accessibility is slightly reduced for the PA/CNT electrodes (7.5\%) and decreased by half for the $(2 \times \mathrm{PA}) / \mathrm{CNT}$ electrodes $(49.8 \%)$, as can be derived from the anodic peak currents (Figure $3 \mathrm{a}$ ). CVs were 

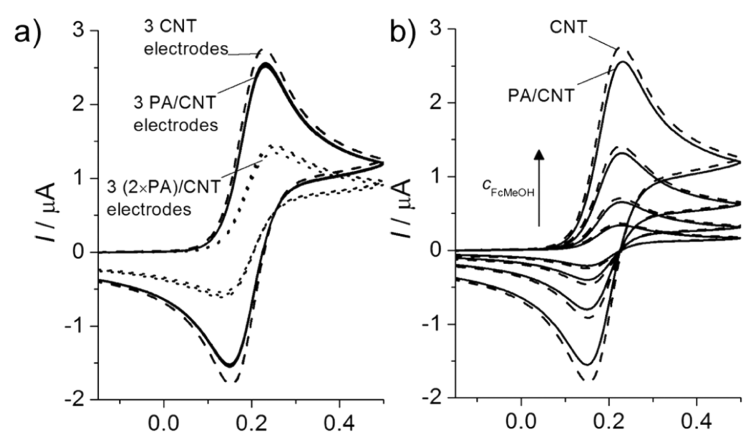

$E / \mathrm{V}$ vs. $\mathrm{Ag} / \mathrm{AgCl} / 1 \mathrm{M} \mathrm{KCl}$
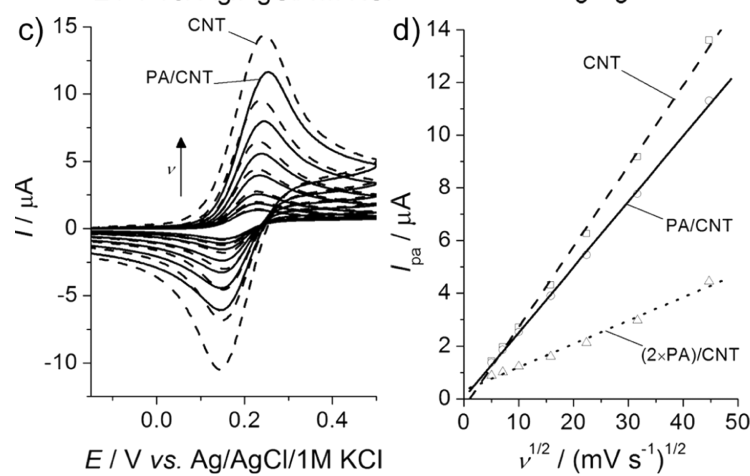

Figure 3. (a) Reproducibility, (b) concentration dependence and (c,d) scan rate dependence of inkjet printed CNT (dashed lines), PA/CNT (solid lines) and $(2 \times \mathrm{PA}) / \mathrm{CNT}$ (dotted lines) electrodes. Electrolyte solution: $\mathrm{FcMeOH}$ and $0.1 \mathrm{M} \mathrm{KCl}$. (a) Three CNT, three PA/CNT and three $(2 \times \mathrm{PA}) / \mathrm{CNT}$ electrodes. $\nu=100 \mathrm{mV} \cdot \mathrm{s}^{-1}, c_{\mathrm{FcMeOH}}=4$ $\mathrm{mM}$. (b) $1 \mathrm{CNT}$ and $1 \mathrm{PA} / \mathrm{CNT}$ electrode. Scan rate $\nu=100 \mathrm{mV} \cdot \mathrm{s}^{-1}$ various $c_{\mathrm{FcMeOH}}$ in $\mathrm{mM}: 0.5,1,2$, and 4 . (c) $1 \mathrm{CNT}$ and $1 \mathrm{PA} / \mathrm{CNT}$ electrode. Various $\nu$ in $\mathrm{mV} \cdot \mathrm{s}^{-1}: 25,50,100,250,500,1000$ and 2000; $c_{\mathrm{FcMeOH}}=4 \mathrm{mM}$. (d) Baseline corrected anodic peak current vs $\nu^{1 / 2}$.

also recorded with various concentrations of $\mathrm{FcMeOH}$ (Figure $3 \mathrm{~b}$ ) and at different scan rates (Figure 3c), confirming the trends discussed above. With increasing hydrogel thickness, larger peak potential separations can be obtained in addition to the smaller peak currents. This is also reflected by the calculated Randles-Sevcik constant, which is close to the theoretical value of 0.4463 for the CNT electrodes but decreases about $18.5 \%$ and $71 \%$ for the PA/CNT and $(2 \times$ $\mathrm{PA} / \mathrm{CNT}$ electrodes, respectively (see Table 1 and the Supporting Information, SI-11). For faster scan rates, the cathodic peak current becomes significantly smaller than the anodic peak current, which might be explained by nonlinear diffusion effects at partially blocked electrodes with high surface coverage of blocking agents (e.g., polymeric chains) as discussed by Amatore et al. ${ }^{41}$ This effect becomes more pronounced for the 4 IJPL hydrogels.
Antioxidant Measurements in Untreated Red Wine and Orange Juice. Electrochemical assays ${ }^{19,43-51}$ for the quantitative measurements of $\mathrm{AOs}$ in liquid samples present several advantages over other methodologies based on, for instance, spectrophotometry ${ }^{52-55}$ or fluorescence microscopy, ${ }^{56}$ as they are simple, fast, can be miniaturized, have a very low sample volume requirement and do not necessarily require sample pretreatment procedures. Voltammetric techniques allow differentiating highly relevant $\mathrm{AOs}$ from less relevant compounds thanks to their lower oxidation potentials and faster reaction kinetics. Carbon based electrodes such as CNTs have demonstrated reliability thanks to their biocompatibility and affinity for organic molecules. We have investigated the properties of the PA/CNT electrodes in comparison to bare $\mathrm{CNT}$ electrodes and CPEs for measuring red wine and orange juice. Red wine $e^{45,50,57-61}$ as well as orange juice ${ }^{62-69}$ have already been studied by several groups using voltammetric analyses, but usually after dilution in order to achieve a linearity between the recorded response and the analyte concentration by reducing the matrix effects. First, GA, one of the most common AOs present in red wine, ${ }^{70}$ was measured by LSV in a $\mathrm{pH} 3$ acetate buffered solution and plotted as the normalized anodic peak currents $I_{\mathrm{pa}} \cdot A_{\mathrm{g}}^{-1}$, where $A_{\mathrm{g}}$ is the geometric electrode area (Figure 4a). Two anodic waves can be observed, which are typical for the electrochemical oxidation of GA. The first peak corresponds to the oxidation of GA into the semiquinone radical and the second one represents the conversion into its quinone form. ${ }^{71,72}$ In comparison to the bare CNT electrode, the PA/CNT electrode shows a slight shift to higher values of the two peak potentials, as well as higher currents for both processes. The latter might be related to the partial protection of the CNT electrode by the PA film against passivation due to the adsorption of oxidation products. Once contaminated, conventional electrode reactivation methods such as mechanical polishing are not applicable for the CPEs and CNT electrodes, because the sensors would be irreversibly damaged. Hence, disposable probes have to be fabricated at low cost and with high reproducibility using batch fabrication processes for the reliable quantitative analysis, in particular, in complex sample matrices. The tested screen printed CPEs showed a relative large variation in their recorded LSV curves in red wine, whereas the inkjet printed CNT and $\mathrm{PA} / \mathrm{CNT}$ electrodes gave more reproducible signals (Figure 4b). Furthermore, the used CNT electrodes show faster reaction kinetics and a catalytic effect, as indicated by a more pronounced slope for the oxidative wave and a shift of the oxidation potentials to lower values, respectively, as reported previously for the monitoring of $\mathrm{AOs}$ in blood transfusion bags. ${ }^{38}$ Generally, three peaks can be observed in the LSVs measured in red wine samples. ${ }^{45,61}$ The first peak $(0.47 \mathrm{~V})$ corresponds to phenolic compounds with ortho-diphenol

Table 1. Parameters of CVs from Figures 3 and S-12 (Supporting Information)

$\begin{array}{lcccc}\text { electrode } & I_{\mathrm{pa}} \times 10^{6} / A^{a} & \text { Randles-Sevcik const. }{ }^{b, c} & b \times 10^{6} / A^{c} & \Delta E_{\mathrm{p}}(\mathrm{mV})^{c} \\ \mathrm{CNT} & 2.711 \pm 0.005 & 0.399 \pm 0.008 & -0.323 \pm 0.150 & 79.9 \pm 10.0 \\ \mathrm{PA} / \mathrm{CNT} & 2.508 \pm 0.022 & 0.325 \pm 0.004 & 0.033 \pm 0.080 & 1.06 \pm 0.15 \\ (2 \times \mathrm{PA}) / \mathrm{CNT} & 1.387 \pm 0.045 & 0.115 \pm 0.005 & 0.320 \pm 0.099 & 1.17 \pm 0.1\end{array}$

${ }^{a}$ Anodic peak currents $I_{\mathrm{pa}}$ measured with three different fresh sensors with $\nu=100 \mathrm{mV} \cdot \mathrm{s}^{-1}$ and $c_{\mathrm{FcMeOH}}=4 \mathrm{mM}$. ${ }^{b}$ Randles - Sevcik const. $=m \cdot\left(n^{3 / 2}\right.$. $\left.A_{\mathrm{g}} \cdot D_{\mathrm{FcMeOH}}{ }^{1 / 2} \cdot c_{\mathrm{FcMeOH}} \cdot R T^{-1 / 2} \cdot F^{3 / 2}\right)^{-1}$, where $F$ is the Faraday constant, $R$ the ideal gas constant, $T$ the temperature, $n$ the number of transferred electrons, $D_{\mathrm{FcMeOH}}=7.8 \cdot 10^{-6} \mathrm{~cm}^{2} \cdot \mathrm{s}^{-1}$ the diffusion coefficient of $\mathrm{FcMeOH}^{42}$ and $A_{\mathrm{g}}$ the geometric area of the electrode. Quantity $m$ was obtained from the curves $I_{\mathrm{pa}}=m \cdot \nu^{1 / 2}+b$. Details in the Supporting Information SI-11. ${ }^{c}$ Obtained from CVs measured with various scan rates. 

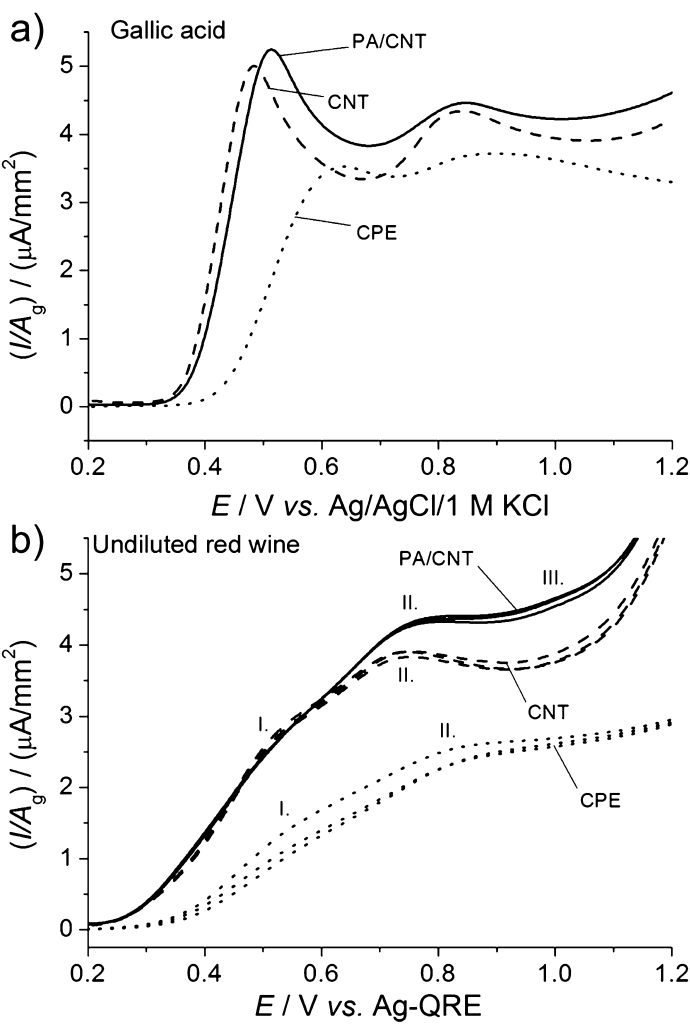

Figure 4. LSVs in (a) $2 \mathrm{mM}$ gallic acid and $0.1 \mathrm{M}$ acetate buffer ( $\mathrm{pH}$ 3 ) and (b) undiluted red wine sample by using PA/CNT electrodes (solid lines), bare CNT electrodes (dashed lines) and CPEs (dotted lines). Each measurement was performed with one fresh sensor. Scan rate $\nu=100 \mathrm{mV} \cdot \mathrm{s}^{-1}$

groups or to $\mathrm{GA}^{73}$ Malvidin anthocyanins contribute to the second peak $(0.73 \mathrm{~V}) .^{73}$ The third peak is usually not easily detected and is caused by the presence of compounds that are more difficult to oxidize. ${ }^{45}$ Indeed, the third peak was only detectable with the PA/CNT electrodes (0.99 V; Figure 4b). The ability to distinguish these three peaks represents clearly another advantage of the ultrathin hydrogel layer.

The higher anodic peak currents for peak II and III observed with the PA/CNT electrodes could be explained by a partial protection of the electrode surface from passivation.

Fresh orange juice represents another source of antioxidants, with AA being its major component with a concentration of around $2.3 \mathrm{mM} .^{63}$ Figure 5a shows LSVs in an acetate buffered AA solution ( $\mathrm{pH}$ 3.8) using the PA/CNT and bare CNT electrodes.

Generally, both electrodes recorded an irreversible oxidation peak of $\mathrm{AA}$ at $0.44 \mathrm{~V}(\mathrm{CNT})$ and $0.53 \mathrm{~V}(\mathrm{PA} / \mathrm{CNT})$, respectively.

The recorded LSVs in untreated orange juice (containing pulp and fibers; $\mathrm{pH}$ 3.8; inset in Figure $5 \mathrm{~b}$ ) showed a significant matrix effect on the CPEs and CNT electrodes responses caused by the composition of the juice (Figure 5b). The normalized anodic peak currents/plateaus $I_{\mathrm{pa}} \cdot A_{\mathrm{g}}{ }^{-1}$ and halfpeak/plateau potentials $E_{\mathrm{pa} / 2}$ were determined from the LSVs and demonstrated that the juice matrix shifts the wave of AA on the CNT electrodes about $186 \mathrm{mV}$ and on CPEs about $257 \mathrm{mV}$ to more positive potentials (Figure $5 \mathrm{~b}$ ). This effect was not obtained in acetate buffered AA solution. By adding the PA film, the nanoenvironment of the CNTs electrode surface is affected, overcoming the matrix effect caused by the untreated
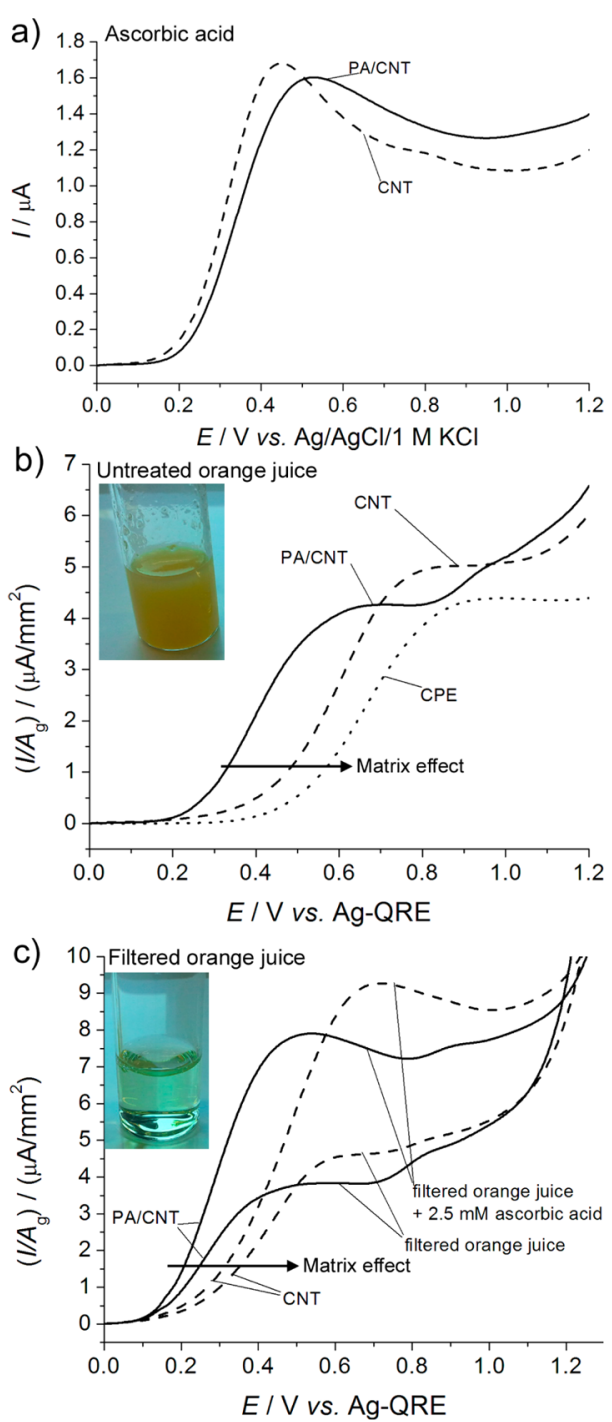

Figure 5. LSVs in (a) $2 \mathrm{mM}$ ascorbic acid and $0.1 \mathrm{M}$ acetate buffer ( $\mathrm{pH}$ 3.8), (b) normalized LSVs in untreated commercial orange juice ( $\mathrm{pH} 3.8)$ and (c) filtered orange juice (200 nm syringe filter; with and without addition of $2.5 \mathrm{mM}$ ascorbic acid) by using PA/CNT electrodes (solid lines), bare CNT electrodes (dashed lines) and CPEs (dotted lines). Scan rate $\nu=100 \mathrm{mV} \cdot \mathrm{s}^{-1}$.

orange juice and leading to the apparition of an additional oxidation peak at $0.96 \mathrm{~V}$. This result might be explained by the PA film that inhibits and filters the strong adsorption of reaction products and other present interfering agents (e.g., pulp and fibers) due to the nanopores (pore size $\sim 13 \mathrm{~nm}$; vide supra) and induced hydrophilic environment. Mechanical filtering of the orange juice using a $200 \mathrm{~nm}$ pore size filter kept the shift of potential (Figure 5c). Moreover, the height of the current plateaus decreased by $10 \%$ and $8 \%$ for the PA/CNT and bare CNT electrodes, respectively. The addition of $2.5 \mathrm{mM}$ AA to the filtered orange juice sample confirmed that the measured signals are mainly due to the oxidation of AA (Figure 5c). Furthermore, the peak current/plateau increased by $106 \%$ and $101 \%$ (in respect to the unfiltered orange juice sample) for the CNT and PA/CNT electrodes, respectively, indicating that the initial concentration of AA in the orange juice sample is in the expected range. The dilution of the orange juice by $1: 10$ resulted in similar LSV curves (Supporting Information, SI-12), which shows that a good electrochemical response is already 
achieved in the initial sample composition where no additional sample treatment (e.g., filtering or dilution of the sample) is required.

\section{CONCLUSION}

A novel strategy to cover reproducibly CNT electrodes with a nanometer thin layer of PA gel by inkjet printing and parallel UV photopolymerization has been demonstrated. One key step of this introduced concept is the prepolymerization of the acrylamide/bis containing ink to adjust the ink viscosity and to enhance the rate of polymerization. Both properties are crucial for fast and reproducible inkjet printing of disposable PA modified CNT electrodes. The existence of a stable and well attached hydrogel has been confirmed by HR SEM and wetting tests. The homogeneously distributed nanopores in the hydrogel layer enable the mass transport of analytes toward the electrode, allowing the usage of the PA/CNT electrodes as amperometric sensors for the direct detection of antioxidants in untreated complex sample matrices. Compared to bare CNT electrodes and CPEs, the hydrogel-coated electrodes compensated for matrix effects in untreated red wine and orange juice samples that are usually only overcome by dilution and filtering. As a result, the voltammetric quantification of the antioxidant power could be achieved free of artifacts caused by matrix effects. ${ }^{68}$ All in all, the PA/CNT electrodes represent a new approach for the amperometric detection of AOs in untreated complex matrices and the PA hydrogel concept brings also new possibilities for the development of functional electrode materials based on UV photopolymerization.

\section{ASSOCIATED CONTENT}

\section{S Supporting Information}

Reaction schemes of the UV photopolymerization, more pictures of jetted droplets, the waveform, stability tests of the $\mathrm{PA} / \mathrm{CNT}$ electrodes, surface dependence, topography measurements of the inkjet printed gel, additional voltammetric experiments and details of the Randles-Sevcik constant calculations. This material is available free of charge via the Internet at http://pubs.acs.org.

\section{AUTHOR INFORMATION}

\section{Corresponding Author}

*H. H. Girault. E-mail: hubert.girault@epfl.ch.

\section{Author Contributions}

The paper was written through contributions of all authors. All authors have given approval to the final version of the paper.

\section{Funding}

This work was supported by the Swiss Commission for Technology and Innovation (CTI, project No. 13841.2PFLSLS).

\section{Notes}

The authors declare no competing financial interest.

\section{ACKNOWLEDGMENTS}

The authors thank Adam Wood, Jim Lamb, Christopher Landorf and Ram Trichur from Brewer Science for the provided information and technical support about the CNT ink. Prof. Dr. Kevin Sivula (EPFL) is thanked for providing the authors the equipment to measure the surface tension of the inks. The Center of Micronanotechnology (CMi) at the EPFL is acknowledged for the access to the Dimatix DMP-2831 printer and technical support.

\section{REFERENCES}

(1) Chrambach, A.; Rodbard, D. Science 1971, 172, 440-451.

(2) Bolto, B.; Gregory, J. Water Res. 2007, 41, 2301-2324.

(3) Wever, D. A. Z.; Picchioni, F.; Broekhuis, A. A. Prog. Polym. Sci. 2011, 36, 1558-1628.

(4) Shen, L.; Huang, R.; Hu, N. Talanta 2002, 56, 1131-1139.

(5) Lu, H.; Li, Z.; Hu, N. Biophys. Chem. 2003, 104, 623-632.

(6) Varma, S.; Mattiasson, B. J. Biotechnol. 2005, 119, 172-180.

(7) Xing, B.; Yin, X.-B. Biosens. Bioelectron. 2009, 24, 2939-2942.

(8) Wu, S.; Tan, W.; Xu, H. Analyst 2010, 135, 2523-2527.

(9) Peng, Y.; Wei, C.-W.; Liu, Y.-N.; Li, J. Analyst 2011, 136, 40034007.

(10) Walcarius, A. Electroanalysis 1998, 10, 1217-1235.

(11) Zen, J.-M.; Kumar, A. S.; Tsai, D.-M. Electroanalysis 2003, 15, 1073-1087.

(12) Yamada, H.; Imahori, H.; Nishimura, Y.; Yamazaki, I.; Ahn, T. K.; Kim, S. K.; Kim, D.; Fukuzumi, S. J. Am. Chem. Soc. 2003, 125, 9129-9139.

(13) Zhang, K.; Zhang, L. L.; Zhao, X. S.; Wu, J. Chem. Mater. 2010, 22, 1392-1401.

(14) Itaya, K. J. Appl. Phys. 1982, 53, 804.

(15) McCreery, R. L. Chem. Rec. 2012, 12, 149-163.

(16) Murray, R. Acc. Chem. Res. 1980, 117, 135-141.

(17) Murray, R.; Ewing, A.; Durst, R. Anal. Chem. 1987, 59.

(18) Arrigan, D. W. M. Analyst 1994, 119, 1953-1966.

(19) Barroso, M. F.; de-los-Santos-Álvarez, N.; Delerue-Matos, C.; Oliveira, M. B. P. P. Biosens. Bioelectron. 2011, 30, 1-12.

(20) Malinauskas, A.; Malinauskiene, J.; Ramanavičius, A. Nanotechnology 2005, 16, R51-62.

(21) Lange, M. A.; Chambers, J. Q. Anal. Chim. Acta 1985, 175, 8997.

(22) Lange, M. A.; Chambers, J. Q. Anal. Chem. 1986, 2874, 28722874.

(23) O'Farrell, P. H. J. Biol. Chem. 1975, 250, 4007-4021.

(24) Issaq, H. J. Electrophoresis 2001, 22, 3629-3638.

(25) Görg, A.; Postel, W.; Westermeier, R. Anal. Biochem. 1978, 89, $60-70$.

(26) Görg, A.; Postel, W.; Westermeier, R.; Gianazza, E.; Righetti, P. G. J. Biochem. Biophys. Methods 1980, 3, 273-284.

(27) Mondal, M. H.; Mukherjee, M.; Kawashima, K.; Nishida, K.; Kanaya, T. Macromolecules 2009, 42, 732-736.

(28) Huang, X.; Doneski, L. J.; Wirth, M. J. Anal. Chem. 1998, 70, 4023-4029.

(29) Xiao, D.; Zhang, H.; Wirth, M. Langmuir 2002, 9971-9976.

(30) Xiao, D.; Le, T. V.; Wirth, M. J. Anal. Chem. 2004, 76, 20552061.

(31) Smith, E. A.; Coym, J. W.; Cowell, S. M.; Tokimoto, T.; Hruby, V. J.; Yamamura, H. I.; Wirth, M. J. Langmuir 2005, 21, 9644-9650.

(32) Tekin, E.; Smith, P. J.; Schubert, U. S. Soft Matter 2008, 4, 703713.

(33) Magdassi, S. The Chemistry of Inkjet Inks; World Scientific Publishing: Singapore, 2010.

(34) Singh, M.; Haverinen, H. M.; Dhagat, P.; Jabbour, G. E. Adv. Mater. 2010, 22, 673-685.

(35) Jensen, G. C.; Krause, C. E.; Sotzing, G. A.; Rusling, J. F. Phys. Chem. Chem. Phys. 2011, 13, 4888-4894.

(36) Liu, X.; Li, T.; Liu, D.; Wang, Z. Anal. Methods 2013, 5, 285.

(37) Wang, M.-W.; Pang, D.-C.; Tseng, Y.-E.; Tseng, C.-C. J. Taiwan Inst. Chem. Eng. 2014, 45, 1049-1055.

(38) Lesch, A.; Cortés-Salazar, F.; Prudent, M.; Delobel, J.; Rastgar, S.; Lion, N.; Tissot, J.-D.; Tacchini, P.; Girault, H. H. J. Electroanal. Chem. 2014, 717-718, 61-68.

(39) Soltman, D.; Subramanian, V. Langmuir 2008, 24, 2224-2231.

(40) de Gans, B.-J.; Schubert, U. S. Langmuir 2004, 20, 7789-7793.

(41) Amatore, C.; Savéant, J. M.; Tessier, D. J. Electroanal. Chem. 1983, 147, 39-51.

(42) Zoski, C. G. Electroanalysis 2002, 14, 1041-1051.

(43) Agui, M. L.; Reviejo, A. J.; Yanez-Sedeno, P.; Pingarron, J. M. Anal. Chem. 1995, 67, 2195-2200. 
(44) Chevion, S.; Roberts, M. A.; Chevion, M. Free Radical Biol. Med.

2000, 28, 860-870.

(45) Kilmartin, P. A.; Zou, H.; Waterhouse, A. L. J. Agric. Food Chem.

2001, 49, 1957-1965.

(46) Brett, A. M. O.; Ghica, M. E. Electroanalysis 2003, 15, 17451750.

(47) Blasco, A. J.; Gonzalez, M. C.; Escarpa, A. Anal. Chim. Acta 2004, 511, 71-81.

(48) Blasco, A. J.; González Crevillén, A.; González, M. C.; Escarpa, A. Electroanalysis 2007, 19, 2275-2286.

(49) Oliveira, R.; Bento, F.; Sella, C.; Thouin, L.; Amatore, C. Anal. Chem. 2013, 85, 9057-9063.

(50) Šeruga, M.; Novak, I.; Jakobek, L. Food Chem. 2011, 124, 12081216.

(51) Sochor, J.; Dobes, J.; Krystofova, O.; Ruttkay-Nedecky, B.; Babula, P.; Pohanka, M.; Jurikova, T.; Zitka, O.; Adam, V.; Klejdus, B.; Kizek, R. Int. J. Electrochem. Sci. 2013, 8, 8464-8489.

(52) Sharma, O. P.; Bhat, T. K. Food Chem. 2009, 113, 1202-1205.

(53) van den Berg, R.; Haenen, G. R. M. M.; van den Berg, H.; Bast,

A. Food Chem. 1999, 66, 511-517.

(54) Miller, N. J.; Rice-Evans, C.; Davies, M. J.; Gopinathan, V.; Milner, A. Clin. Sci. 1993, 84, 407-412.

(55) Benzie, I. F.; Strain, J. J. Anal. Biochem. 1996, 239, 70-76.

(56) Cao, G.; Alessio, H.; Cutler, R. Free Radical Biol. Med. 1993, 14, 303-311.

(57) Zou, H.; Kilmartin, P. A.; Inglis, M. J.; Frost, A. Aust. J. Grape. Wine Res. 2002, 8, 163-174.

(58) Piljac, J.; Martinez, S.; Stipčević, T.; Petrović, Ž.; MetikošHuković, M. Am. J. Enol. Vitic. 2004, 55, 417-422.

(59) Parra, V.; Arrieta, Á. A.; Fernández-Escudero, J.-A.; RodríguezMéndez, M. L.; De Saja, J. A. Sens. Actuators, B 2006, 118, 448-453.

(60) Roginsky, V.; de Beer, D.; Harbertson, J. F.; Kilmartin, P. A.; Barsukova, T.; Adams, D. O. J. Sci. Food Agric 2006, 86, 834-840.

(61) Rebelo, M. J.; Rego, R.; Ferreira, M.; Oliveira, M. C. Food Chem.

2013, 141, 566-573.

(62) Pournaghi-Azar, M.; Ojani, R. Talanta 1997, 9140.

(63) Miller, N. J.; Rice-Evans, C. A. Food Chem. 1997, 60, 331-337.

(64) Amorini, A. M.; Fazzina, G.; Lazzarino, G.; Tavazzi, B.; Pierro, D. D.; Santucci, R.; Sinibaldi, F.; Galvano, F.; Galvano, G. Free Radical Res. 2001, 35, 953-966.

(65) Sousa, W. R.; da Rocha, C.; Cardoso, C. L.; Silva, D. H. S.; Zanoni, M. V. B. J. Food Comp. Anal. 2004, 17, 619-633.

(66) Pisoschi, A. M.; Pop, A.; Negulescu, G. P.; Pisoschi, A. Molecules 2011, 16, 1349-1365.

(67) Kumar, S.; Vicente-Beckett, V. Beilstein J. Nanotechnol. 2012, 3, $388-396$.

(68) Tacchini, P.; Lesch, A.; Neequaye, A.; Lagger, G.; Liu, J.; CortésSalazar, F.; Girault, H. H. Electroanalysis 2013, 25, 922-930.

(69) Wang, X.; Jiao, C.; Yu, Z. Sens. Actuators, B 2014, 192, 628-633.

(70) Lachman, J.; Šulc, M.; Faitová, K.; Pivec, V. Int. J. Wine Res. 2009, 1, 101-122.

(71) Gunckel, S.; Santander, P.; Cordano, G.; Ferreira, J.; Munoz, S.; Nunez-Vergara, L. J.; Squella, J. A. Chem.-Biol. Interact. 1998, 114, 4559.

(72) Souza, L. P.; Calegari, F.; Zarbin, A. J. G.; Marcolino-Junior, L. H.; Bergamini, M. F. J. Agric. Food Chem. 2011, 59, 7620-7625.

(73) Kilmartin, P. A.; Zou, H.; Waterhouse, A. L. Am. J. Enol. Vitic. 2002, 53, 294-302. 